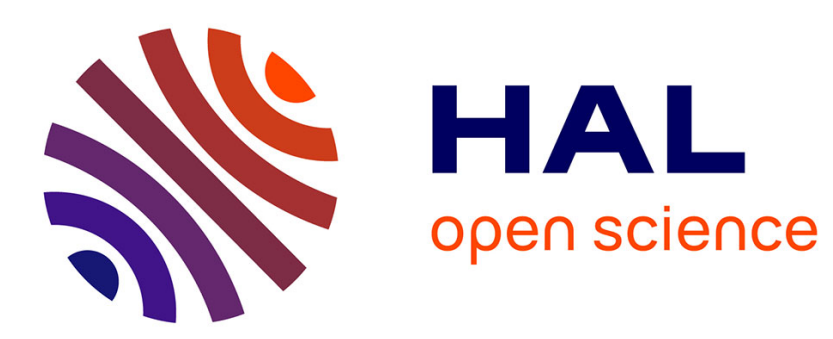

\title{
Cell interactions and cell signaling during hematopoietic development
}

\author{
C Drevon, Thierry Jaffredo
}

\section{To cite this version:}

C Drevon, Thierry Jaffredo. Cell interactions and cell signaling during hematopoietic development. Experimental Cell Research, 2014, 329 (2), pp.200 - 206. 10.1016/j.yexcr.2014.10.009 • hal-01090559

\section{HAL Id: hal-01090559 \\ https://hal.science/hal-01090559}

Submitted on 3 Dec 2014

HAL is a multi-disciplinary open access archive for the deposit and dissemination of scientific research documents, whether they are published or not. The documents may come from teaching and research institutions in France or abroad, or from public or private research centers.
L'archive ouverte pluridisciplinaire HAL, est destinée au dépôt et à la diffusion de documents scientifiques de niveau recherche, publiés ou non, émanant des établissements d'enseignement et de recherche français ou étrangers, des laboratoires publics ou privés. 


\title{
Cell interactions and cell signaling during hematopoietic development.
}

\author{
C. Drevon ${ }^{1,2} \&$ T. Jaffredo ${ }^{1,2,3}$ \\ ${ }^{1}$ Sorbonne Universités, UPMC Univ Paris 06, IBPS, UMR 7622, Laboratoire de Biologie du Développement, \\ 75005 Paris \\ ${ }^{2}$ CNRS, UMR 7622, Inserm U 1156, IBPS, Laboratoire de Biologie du Développement, 75005 Paris \\ ${ }^{3}$ Corresponding author. E-mail : thierry.jaffredo@upmc.fr
}

\begin{abstract}
Hematopoiesis is a key process that leads to the formation of all blood cell lineages from a specialized, multipotent cell, named the Hematopoietic Stem Cell (HSC). During development, the embryo produces several waves of hematopoiesis that produce specialized subsets of hematopoietic cells. Tissue interactions and cell signaling play an essential role in developmental hematopoiesis by allowing the formation of hematopoietic and endothelial cells (EC) from the mesoderm in particular in the yolk sac and by instructing the different generations of hematopoietic cells (HC). The embryonic aorta is another site wherein tissue interaction is essential for the production of the first HSCs that is achieved from a specialized subset of hemogenic endothelial cells. This production is tightly time- and space-controlled with the transcription factor Runxl and the Notch signaling pathway playing a key role in this process and the surrounding tissues controlling the aortic shape and fate. Here we shall briefly review how hemogenic EC differentiate from the mesoderm, how the different aortic components assemble coordinately to establish the dorso-ventral polarity resulting in the initiation of Runxl expression in hemogenic EC and the initiation of the hematopoietic program through modulation of the NotchRunx1 axis. These data should help elucidate the first steps in HSC commitment and bring further insights into the manipulation of adult HSCs.
\end{abstract}

Key words: Chicken, quail, mouse, chimeras, development, endoderm, hematopoietic stem cells, aorta, endothelium, Notch.

\section{Introduction}

Hematopoietic stem cells (HSCs) are specialized multipotent stem cells that emerge during early embryogenesis, whose functions are to maintain hematopoiesis throughout the entire lifespan of the organism. In the adult, HSCs are located in the bone marrow where they self-renew and differentiate to give rise to all blood cell lineages. However, despite at least two decades of intense investigations, their embryological origin remains controversial. It is well accepted that embryonic and fetal hematopoiesis are separated into three waves that occur at different times and locations. The first wave takes place very early during development i.e. Embryonic day (E) 7.25 [1] for the mouse embryo and at 19-22 hours of incubation for the chick [2,3]. This wave gives rise to primitive erythroid cells, macrophages and megakaryocytes. From E8.9 in the mouse i.e. shortly after the onset of primitive hematopoiesis, the first definitive (adult-type) hematopoietic cells emerge from the yolk sac. These cells are definitive erythroid and myeloid progenitors but a recent report indicates that an immune- 
restricted, lymphoid-primed progenitor also emerges in the yolk sac before colonizing the fetal liver [4]. Similar erythroid and myeloid progenies were shown to take place in the chick yolk sac at a slightly different time between E6 and E12 [5]. The third wave occurs in the embryo and is characterized by the production of HSCs and adult-type erythromyeloid progenitors from ECs of the aorta and associated arteries through the stereotyped production of intra-aortic hematopoietic cell clusters (IAHC). It was shown however that both cell types originate from distinct types of endothelium [6] suggesting the existence of specialized subsets of EC and/or microenvironments.

In this review, we will focus on some aspects of cell-cell interactions that appear to be the hallmarks of embryonic hematopoietic cell emergence.

\section{The close proximity between endothelial and hematopoietic cells:}

In vertebrates, the first elements of the blood-forming system differentiate from the mesoderm during gastrulation. The original observation that hematopoietic and endothelial lineages differentiated simultaneously in the yolk sac to form blood islands $[7,8]$ led to the hypothesis that both cell types were generated from a common mesodermal precursor, the hemangioblast [8]. The yolk sac blood islands wherefrom the first $\mathrm{HC}$ emerge are indeed constituted of and outer layer of EC an a core of $\mathrm{HC}$. The possible common origin of both cell types constituted a biological enigma that is not completely solved after a quarter century of investigations. Studies on embryonic stem cells reveal that hematopoietic and vascular commitment can be completely recapitulated in vitro using controlled culture systems. These studies were also instrumental to demonstrate that hematopoietic and endothelial precursors can develop from the same cell $[9,10]$ hence revealing the existence of the hemangioblast postulated half a century ago.

The fact that the AGM region harbors IAHC tightly associated to the aortic endothelium was suggestive of an aorta-borne hematopoietic production by specialized EC, qualified as hemogenic. This idea was reinforced by the observation that $\mathrm{EC}$ and $\mathrm{HC}$ share common markers i.e., CD34, VE cadherin, and by the fact that Flk-1 and Tie2 knock-out mice showed defects in both hematopoietic and endothelial lineages [11-14]. Using lineagetracing experiments in chicks $[15,16]$ or in mice [17-19] IAHC were shown to derive from the endothelium through a dynamic process called endothelial-to-hematopoietic transition (EHT). Recently, live imaging technology in vitro on ES cells [20,21] and in vivo on living embryos or tissues [22-25] allowed visualizing the de novo production of phenotypically defined HSC or progenitor cells from the aortic endothelium.

\section{The crucial role of the endoderm:}

Interactions between neighboring tissue are required for developmental patterning. The endoderm has long been proposed to play an early role during commitment of the blood-forming system. By separating the hypoblast (primitive endoderm) from the yolk sac mesoderm and ectoderm at an early developmental stage, Wilt was among the first to show that endoderm was dispensable, albeit stimulatory, for erythropoietic formation to occur [26, 27] but was absolutely required for EC commitment [26]. A key molecule of erythropoietic commitment was shown to be basic Fibroblast Growth Factor (bFGF) produced by the hypoblast [28]. In agreement with these results, M. Baron and co-workers reported that early hematopoiesis in mouse embryo was 
not mesoderm autonomous, but required contact or signals released from the visceral endoderm. They identified Indian Hedgehog as expressed by the visceral endoderm of the gastrulating embryo and showed that the Indian Hedgehog protein was able to activate hematopoiesis in pre or early gastrulating epiblast in the absence of visceral endoderm [29]. Interestingly in the chick embryo, Indian Hedgehog is regionalized and expressed exclusively in the yolk sac endoblast, the mouse equivalent of the visceral endoderm (TJ personal communication); furthermore, the dynamic expression of Gata2 during the onset of yolk sac erythropoiesis, showed that this factor is expressed very early during gastrulation in groups of cells, likely to represent the first endothelial precursors, in close association with the endoderm [3]. These first endothelial precursors are formed under the control of a VEGF-VEGF-R2 axis that triggers expression the SCL-Tal1 transcription factor and the subsequent commitment into the endothelial lineage [30]. In addition to endothelial precursors, SCL-Tal1 was shown to be regulated by a transcription factor complex that consists of Fli-1, Elf-1, two ETS- related transcription factors and Gata-2 [31]. In addition Fli-1 was shown to sit at the top of the hematopoietic hierarchy being able to activate the expression of SCL-Tal-1, Lmo2, Gata-2 and Flk1 [32]. Of note Scl-Tal-1, Fli-1, and Gata2 have been shown to form a transcriptional complex which directly regulates the key hematopoietic player Runx1 [33].

In the embryo proper, production of IAHC is restricted to the ventral part of the fused dorsal aorta, whose origin is splanchnopleural [34]. The avian embryo model has been instrumental in demonstrating the cellular and molecular bases of this dorso-ventral patterning. Such regionalization was explained by demonstrating the dual origin of embryonic EC. One originates from the somite and subsequently colonizes the somatopleural mesoderm. Somite-derived EC give rise to the vasculature of the body wall and limbs and are devoid of hemogenic capacity. Another EC lineage originates from the splanchnopleural mesoderm, vascularize the visceral organs and display hemogenic capacities. Interestingly non-hemogenic, somite-derived EC can be converted into hemogenic EC by treatment with either VEGF, FGF, TGFß or contact with the endoderm [35]. Thus, it is likely that the hemogenic endothelium of the aorta is formed following exposure to at least one or a combination of these factors present in the embryonic endoderm and/or mesoderm. In addition to the above-cited factors Sonic Hedgehog, another member of the Hedgehog family is specifically expressed in the embryonic, i.e., visceral in the mouse, endoderm at the exception of its medial-most part located immediately underneath the notochord [36] and (TJ unpublished results) and plays a role in tubule formation [36]. Sonic Hedgehog, present in the intestinal endoderm, immediately underneath the ventral side of the hematopoietic aorta was also suggested to play a trophic role in HSC induction and the formation of a dorso-ventral gradient in the aorta [37].

\section{Control of runx1 expression and intra-aortic cluster formation by the sub-aortic mesoderm.}

Runx1 is a transcription factor shown to be a key regulator of EHT during aortic hematopoiesis $[18,38]$. Runx1 inactivation in the mouse embryo results in the absence of IAHC [22,39] and fetal liver HSCs [40] with a death at E12.5 albeit primitive hematopoiesis is preserved. Runx1 deficiency in embryonic stem cells also prevents the formation of $\mathrm{HC}$ from hemogenic EC [21]. In the chick embryo, we showed that runxl expression was spatially and temporally controlled during the course of definitive hematopoiesis [34]. Runxl expression, restricted to the EC layer in the avian species, initiated around 50h of incubation in the lateral part of the paired aortas shortly followed by c-myb and pul. It then spread latero-ventrally to become expressed by the whole 
ventral endothelium and the IAHC at 70h of incubation. This lateral-to-ventral progression was suggestive of runxl induction by adjacent cells or tissues. It also reveals that the aortic endothelium was primed to respond to this induction. The sub-aortic mesenchyme, closely associated to EC of the aortic floor appeared as a likely candidate. Fate mapping experiments with DiI (a lipophilic dye) crystals on cultured chick embryos showed that both the sub-aortic mesenchyme and the primary aortas originated from the splanchnopleural mesoderm. By making a slit on one side of the embryo between the somites and the intermediate mesoderm to prevent mesoderm ingression underneath the aorta, we were able to prevent the formation of the sub-aortic mesenchyme. The slit did not impair vessel formation nor arterial identity, as shown by ve-cadherin and delta like 4 expression, but blocked the initiation of runxl expression and the subsequent formation of the IAHC. These results clearly showed that the sub-aortic mesenchyme is a key element required for proper initiation of aortic hematopoiesis. However it did not rule out the possibility that the sub-aortic mesenchyme was carrying HC that would subsequently form IAHC. To answer this question, we specifically labeled lateral plate mesoderm by inoculation into the coelom of a dye, CFDA-SE (carboxyfluorescein diacetate n-succinimidyl ester) one day before the formation of IAHC. At the stage of inoculation, the coelom is delineated by two epithelial sheets namely the somatopleural mesoderm and the splanchnopleural mesoderm that will respectively form the mesoderm of the body wall and the mesoderm associated to visceras. CFDA-SE is a lipophilic dye that has been used in a variety of studies to label different tissue layers. This highly fluorescent compound enters cells by diffusion and is cleaved by intracellular enzymes into a fluorescent, non-diffusible product, unable to leave the cell. CFDA-SE has been reported not to be able to permeate through epithelial barriers thus we used it to tag lateral plate derivatives but not the newly formed ventral endothelial network already segregated from the splanchopleural mesoderm at the stage of inoculation thus ensuring a separation of the two lineages. When analyzed at the time of aortic hematopoiesis, the sub-aortic mesenchyme was found labeled but IAHC were free of CFDA-SE, indicating that the sub-aortic mesenchyme did not harbor the forerunners of the IAHC [34].

\section{Notch signaling and aortic hematopoiesis}

The Notch pathway delivers paracrine signals between neighboring cells and is known to be of major importance in cell communication and binary cell fate decision. Notch signaling has been shown to be required in many steps of vascular formation, including arterial specification, and long-term definitive hematopoiesis in zebrafish and mice embryos in a context-dependent manner. In the absence of Notch, definitive hematopoiesis and HSC development, but not primitive hematopoiesis, was impaired [41, 42]. If it has been clearly established that Gata2, but not Runxl, was directly controlled by the Notch-Jagged axis [43] how and when Notch plays a role in the initiation of aortic hematopoiesis from the hemogenic endothelium, remained to be determined.

Since EHT crucially involves a cell fate change, we postulated that Notch signaling should play a role in the process. We thus investigated in detail the changes that took place in the Notch signaling pattern during the initiation of runxl expression in the aorta and the subsequent formation of intra-aortic HC clusters using precise gene expression analysis on embryo section and quantitative RT-PCR on EC and HC populations purified by flow cytometry. 
Several actors of the Notch pathway are present in the EC layer and the IAHC during aortic hematopoiesis [44]. However because the Notch-Ligand axis is implicated in multiple steps in different cell types during aortic hematopoiesis, little is known about the precise requirements of this pathway during EHT. Because the chicken embryo is an appropriate model to investigate discrete steps during aorta formation, we investigated the role of Notch signaling during the early steps of aortic hematopoiesis in this model. We focused on notchl and on the avian orthologues of jaggedl, jagged 2 and delta-like 4 when the aortic floor expressed runxl. We showed that, at the time of aortic fusion, jagged1, 2 and delta-like 4 mRNAs were expressed throughout all aortic EC including those of the floor. Following fusion, runxl expression intensified and jaggedl, 2 and delta-like 4 expression progressively disappeared from the floor precisely where runxl expression was the highest. Finally as IAHC differentiated, they formed in the absence of jaggedl, 2 and delta-like 4 mRNA expression and at least in the absence of jaggedl protein. The same pattern is observed in the human embryo at E35 (Canto and Jaffredo, unpublished results). To further investigate the modulation of the Notch pathway during aortic hematopoiesis, we performed Qantitative RT-PCR experiments on purified endothelial and hematopoietic populations at different steps of their maturation on both chicken and mouse models. Both embryo species displayed a similar expression pattern of Notch signaling in EC at the pre-hematopoietic stage with a prominent expression of Notch1, Jagged1, 2, Delta-like 4 and Hesl (a canonical Notch target). The ECassociated expression pattern remained unchanged at the time of $\mathrm{HC}$ production, but expression of Notch ligands (Jagged1, 2, and Delta-like 4) strongly decreased together with an increase in RbpjK expression in HC as compared to EC. Although changes were more visible in the chicken embryo, both species followed the same pattern, except for Jagged 2 in the mouse embryo, which showed no decrease in $\mathrm{HC}$ relative to EC in agreement with a critical role demonstrated for Jaggedl but not for Jagged2 [45] during aortic hematopoiesis in this species. This decrease in Notch signaling was confirmed using a Runx1-GFP reporter mouse [46] to analyze Hes1 expression in purified populations of hemogenic endothelium (Runx1-GFP ${ }^{+} \mathrm{CD} 144^{+} \mathrm{CD} 41^{-} \mathrm{CD} 45^{-}$), immature HC (Runx1-GFP ${ }^{+} \mathrm{CD} 144^{+} \mathrm{CD} 41^{+} \mathrm{CD} 45^{-}$), and two more mature $\mathrm{HC}$ populations (Runx1$\mathrm{GFP}^{+} \mathrm{CD} 144^{+} \mathrm{CD} 41^{+} \mathrm{CD} 45^{+}$and Runx1-GFP ${ }^{+} \mathrm{CD} 144^{+} \mathrm{CD} 41^{-} \mathrm{CD} 45^{+}$) at the $40-45$ somite pair stage (E11.5). All three HC populations showed reduced levels of Hesl expression relative to ECs. Together, these data suggest that Notch signaling is reduced in HC relative to hemogenic EC and that decreased still further as HC matured in the aorta. Finally when we tracked Notch activity using a reporter plasmid, we concluded that Notch was expressed in EC and in the sub-aortic mesenchyme whereas EHT is accompanied by a decrease in Notch activation and more generally in Notch signaling.

\section{Insights into BMP/TGFB signaling}

BMP signaling is a key event during ventral mesoderm commitment and its subsequent induction into hematopoietic cells. In zebrafish [47], mouse [48] or human [49] embryos, BMP4 is expressed in the mesenchyme underlying the aorta and was shown to be required for HSC maintenance [48]. Wilkinson et al showed that Bmp4 is required for HSC emergence in the ventral wall of the zebrafish aorta and contributes, along with Sonic Hedgehog expressed dorsally, to polarize the vessel [47]. BMP4 thus looked as a good candidate to control or promote aortic hematopoiesis. 
BMPs are members of the BMP/TGF- $\beta$ superfamily that bind type II and type I serine-threonine kinase receptors, and transduce signals through Smad and non-Smad signaling pathways [50]. Aiming to characterize the $\mathrm{BMP} / \mathrm{TGF} \beta$ requirements for hematopoietic emergence in the aorta, we undertook a systematic analysis of the BMP/TGFß pathway in flow cytometry-sorted ECs and HCs of the mouse and chicken aorta using quantitative PCR, in situ hybridization or immunohistochemistry and functional validation using Dorsomorphin, a chemical inhibitor of Smad phosphorylation. We found that expression of Alk1, BmpR2 and Endoglin as type I, II and III receptors respectively characterize the endothelium. This association is typical of TGFß-mediated signaling and indeed the ligand was also found expressed in the endothelium indicating a self-autonomous mechanism. Smad6 and, to a less extent Smad 7 were also found significantly increased in the endothelial fraction indicating a tight control of the pathway as previously reported [51]. Interestingly the presence of a BMP-specific signaling pathway was less prominent. The presence of a strong phosphorylated Smad1, 5, 8 signal in the endothelium together with Alk1 and BmpR2 is in keeping with an early role of TGFß during angioblast migration and vessel formation [52]. This endothelial signature persists at the time of hematopoiesis. Hematopoietic formation was accompanied by a strong decrease of the TGFß pathway without any significant increase in the BMP pathway. Indeed hematopoietic clusters were devoid of any TGFß or BMP-specific phosphorylated Smads and Alk1 expression was lost in IAHC both at the mRNA and protein levels indicating a collapse in the BMP/TGFß pathway is required for hematopoiesis to proceed.

Treatment of the embryos with dorsomorphin, an inhibitor of Smad phosphorylation, significantly promotes hematopoietic production in chick embryos. Taken together, these results indicate that inhibition of the BMP/TGFß pathway is a critical event in the endothelial-to-hematopoietic transition and the subsequent formation of aortic hematopoiesis (submitted).

\section{Conclusion.}

The cellular mechanisms by which hematopoietic (stem) cells are generated during embryonic development are quite well understood; however the molecular events that precisely control spatial and temporal cell emergence remain unclear. The aorta microenvironment is likely to be the source of a cross talk between different signaling pathways that contribute to its regionalized pattern and provide the proper signals to the committed splanchnopleural endothelium. Here we have disclosed a temporally restricted decrease of Notch and BMP/TGFß signaling to initiate hematopoiesis from the hemogenic endothelium. This has been possible, thanks to the avian model that allows identification of discrete steps in the EHT process but also to the comparison of avian and mouse species, which allows to cross-validate the expression patterns and to identify mechanisms, conserved between species.

\section{Acknowledgments.}

The authors wish to thank all members of the laboratory, past and present, for their contributions. Sophie Gournet is acknowledged for drawing assistance. Work was supported by the Centre National de la Recherche Scientifique, the University Pierre \& Marie Curie, and by grants from the Fondation pour la Recherche Médicale (DEQ20100318258) and ANR/CIRM joint grant (ANR/CIRM 0001-02). 


\section{Figure Legend}

Developmental history of the aorta, its EC and HC-associated gene lineages and expression of the Notch pathway.

Boxes lateral to the drawings indicate the expression of the Notch pathway as well as that of hematopoieticspecific genes. The size of the letters stipulates the relative levels of mRNA expression.

A. Early formation of the vascular network. Angioblast (orange) commitment has occurred close to the endoderm and the splanchnopleural mesoderm (pink) but lumenization has not taken place yet.

B. Before fusion of the aortic anlagen. Aortic EC (orange) derive from the splanchnopleural mesoderm. As the embryo develops, the splanchnopleural mesoderm is folding and comes close to the lateral part of the aortic rudiment.

C. Paired aorta stage. Folding of the splanchnopeural mesoderm around the aorta proceeds. Splanchnopleurederived cells insinuate between the ventral aspect of the aorta and the endoderm, separating the two tissues. Runxl becomes expressed in EC (red) having contact with the underlying mesenchyme. The somite provides EC (yellow) that are going to migrate to the body wall and the aorta.

D. Immediately before fusion. Cells of the splanchnopleural mesoderm keep on separating the ventral aspect of the aorta from the endoderm. ECs of the initial roof (splanchnopleural origin, orange) have been replaced by ECs from the somite (yellow). Cells contacting the splanchnopleural mesoderm now express runx1 (red) and initiate $c-m y b$ and $p u-1$ expression.

E. Single aorta stage. Ventral (runxl-positive, red) and dorsal (runxl-negative, yellow) EC populations are represented. The lateral-most hemogenic EC begin to undergo EHT and thickened.

G. Hematopoietic stage. Same legend as in E except that budding HC clusters are now present.

Legends : Ao : aorta ; En : endoderm; N : notochord; NT : neural tube ; S : somite ; So : somatopleural mesoderm ; Sp : splanchnopleural mesoderm.

\section{References.}

[1] J. Palis, S. Robertson, M. Kennedy, C. Wall, G. Keller, Development of erythroid and myeloid progenitors in the yolk sac and embryo proper of the mouse, Development 126 (1999) 5073-5084.

[2] G. Sheng, Primitive and definitive erythropoiesis in the yolk sac: a bird's eye view, Int J Dev Biol 54 (2010) 1033-1043.

[3] K. Minko, K. Bollerot, C. Drevon, M.F. Hallais, T. Jaffredo, From mesoderm to blood islands: patterns of key molecules during yolk sac erythropoiesis, Gene Expr Patterns 3 (2003) 261-272.

[4] C. Boiers, J. Carrelha, M. Lutteropp, S. Luc, J.C. Green, E. Azzoni, P.S. Woll, A.J. Mead, A. Hultquist, G. Swiers, E.G. Perdiguero, I.C. Macaulay, L. Melchiori, T.C. Luis, S. Kharazi, T. Bouriez-Jones, Q. 
Deng, A. Ponten, D. Atkinson, C.T. Jensen, E. Sitnicka, F. Geissmann, I. Godin, R. Sandberg, M.F. de Bruijn, S.E. Jacobsen, Lymphomyeloid Contribution of an Immune-Restricted Progenitor Emerging Prior to Definitive Hematopoietic Stem Cells, Cell Stem Cell (2013).

[5] P.T. Guedes, B.C. de Oliveira, P.P. Manso, L.F. Caputo, G. Cotta-Pereira, M. Pelajo-Machado, Histological analyses demonstrate the temporary contribution of yolk sac, liver, and bone marrow to hematopoiesis during chicken development, PLoS One 9 (2014) e90975.

[6] M.J. Chen, Y. Li, M.E. De Obaldia, Q. Yang, A.D. Yzaguirre, T. Yamada-Inagawa, C.S. Vink, A. Bhandoola, E. Dzierzak, N.A. Speck, Erythroid/myeloid progenitors and hematopoietic stem cells originate from distinct populations of endothelial cells, Cell Stem Cell 9 (2011) 541-552.

[7] F.R. Sabin, Studies on the origin of blood vessels and of red corpuscles as seen in the living blastoderm of the chick during the second day of incubation., Contributions to Embryology 9 (1920) 213-262.

[8] P.D.F. Murray, The development "in vitro" of blood of the early chick embryo., Strangeways Res. Lab. Cambridge. (1932) 497-521.

[9] K. Choi, M. Kennedy, A. Kazarov, J.C. Papadimitriou, G. Keller, A common precursor for hematopoietic and endothelial cells, Development 125 (1998) 725-732.

[10] M. Kennedy, M. Firpo, K. Choi, C. Wall, S. Robertson, N. Kabrun, G. Keller, A common precursor for primitive erythropoiesis and definitive haematopoiesis, Nature 386 (1997) 488-493.

[11] F. Shalaby, J. Ho, W.L. Stanford, K.-D. Fischer, A. Schuh, L. Schwartz, A. Bernstein, J. Rossant, A requirement for $f l k-1$ in primitive and definitive hematopoiesis and vasculogenesis., Cell 89 (1997) 981990.

[12] F. Shalaby, J. Rossant, T.P. Yamaguchi, M. Gertsenstein, X.-F. Wu, M.L. Breitman, A.C. Schuh, Failure of blood-island formation and vasculogenesis in Flk-1-deficient mice., Nature 376 (1995) 6266.

[13] D.J. Dumont, G. Gradwohl, G.H. Fong, M.C. Puri, M. Gertsenstein, A. Auerbach, M.L. Breitman, Dominant-negative and targeted null mutations in the endothelial receptor tyrosine kinase, tek, reveal a critical role in vasculogenesis of the embryo, Genes Dev 8 (1994) 1897-1909.

[14] N. Takakura, X.L. Huang, T. Naruse, I. Hamaguchi, D.J. Dumont, G.D. Yancopoulos, T. Suda, Critical role of the TIE2 endothelial cell receptor in the development of definitive hematopoiesis, Immunity 9 (1998) 677-686.

[15] T. Jaffredo, R. Gautier, V. Brajeul, F. Dieterlen-Lievre, Tracing the progeny of the aortic hemangioblast in the avian embryo, Dev Biol 224 (2000) 204-214.

[16] T. Jaffredo, R. Gautier, A. Eichmann, F. Dieterlen-Lievre, Intraaortic hemopoietic cells are derived from endothelial cells during ontogeny, Development 125 (1998) 4575-4583.

[17] A.C. Zovein, J.J. Hofmann, M. Lynch, W.J. French, K.A. Turlo, Y. Yang, M.S. Becker, L. Zanetta, E. Dejana, J.C. Gasson, M.D. Tallquist, M.L. Iruela-Arispe, Fate tracing reveals the endothelial origin of hematopoietic stem cells, Cell Stem Cell 3 (2008) 625-636.

[18] M.J. Chen, T. Yokomizo, B.M. Zeigler, E. Dzierzak, N.A. Speck, Runx1 is required for the endothelial to haematopoietic cell transition but not thereafter, Nature 457 (2009) 887-891.

[19] M. de Bruijn, X. Ma, C. Robin, K. Ottersbach, M.J. Sanchez, E. Dzierzak, Hematopoietic stem cells localise to the endothelial cell layer in the midgestation mouse aorta., Immunity 16 (2002) 673-683.

[20] H.M. Eilken, S. Nishikawa, T. Schroeder, Continuous single-cell imaging of blood generation from haemogenic endothelium, Nature 457 (2009) 896-900.

[21] C. Lancrin, P. Sroczynska, C. Stephenson, T. Allen, V. Kouskoff, G. Lacaud, The haemangioblast generates haematopoietic cells through a haemogenic endothelium stage, Nature 457 (2009) 892-895.

[22] K. Kissa, P. Herbomel, Blood stem cells emerge from aortic endothelium by a novel type of cell transition, Nature 464 (2010) 112-115.

[23] J.Y. Bertrand, N.C. Chi, B. Santoso, S. Teng, D.Y. Stainier, D. Traver, Haematopoietic stem cells derive directly from aortic endothelium during development, Nature 464 (2010) 108-111.

[24] E.Y. Lam, C.J. Hall, P.S. Crosier, K.E. Crosier, M.V. Flores, Live imaging of Runx1 expression in the dorsal aorta tracks the emergence of blood progenitors from endothelial cells, Blood 116 (2010) 909914.

[25] J.C. Boisset, W. van Cappellen, C. Andrieu-Soler, N. Galjart, E. Dzierzak, C. Robin, In vivo imaging of haematopoietic cells emerging from the mouse aortic endothelium, Nature 464 (2010) 116-120.

[26] F. Wilt, H., Erythropoiesis in the chick embryo : the role of the endoderm., Science 147 (1965) 15881590.

[27] J. Kessel, B. Fabian, Inhibitory and stimulatory influences on mesodermal erythropoiesis in the early chick blastoderm, Development 101 (1987) 45-49.

[28] C. Gordon-Thomson, B.C. Fabian, Hypoblastic tissue and fibroblast growth factor induce blood tissue (haemoglobin) in the early chick embryo, Development 120 (1994) 3571-3579. 
[29] M.A. Dyer, S.M. Farrington, D. Mohn, J.R. Munday, M.H. Baron, Indian hedgehog activates hematopoiesis and vasculogenesis and can respecify prospective neurectodermal cell fate in the mouse embryo, Development 128 (2001) 1717-1730.

[30] P.B. Giles, C.L. Candy, P.A. Fleming, R.W. Owens, W.S. Argraves, C.J. Drake, VEGF directs newly gastrulated mesoderm to the endothelial lineage, Dev Biol 279 (2005) 169-178.

[31] B. Gottgens, A. Nastos, S. Kinston, S. Piltz, E.C. Delabesse, M. Stanley, M.J. Sanchez, A. Ciau-Uitz, R. Patient, A.R. Green, Establishing the transcriptional programme for blood: the SCL stem cell enhancer is regulated by a multiprotein complex containing Ets and GATA factors, Embo J 21 (2002) 3039-3050.

[32] F. Liu, M. Walmsley, A. Rodaway, R. Patient, Fli1 acts at the top of the transcriptional network driving blood and endothelial development, Curr Biol 18 (2008) 1234-1240.

[33] A. Ciau-Uitz, L. Wang, R. Patient, F. Liu, ETS transcription factors in hematopoietic stem cell development, Blood Cells Mol Dis 51 (2013) 248-255.

[34] C. Richard, C. Drevon, P.Y. Canto, G. Villain, K. Bollerot, A. Lempereur, M.A. Teillet, C. Vincent, C. Rossello Castillo, M. Torres, E. Piwarzyk, N.A. Speck, M. Souyri, T. Jaffredo, EndothelioMesenchymal Interaction Controls runx1 Expression and Modulates the notch Pathway to Initiate Aortic Hematopoiesis, Dev Cell 24 (2013) 600-611.

[35] L. Pardanaud, F. Dieterlen-Lievre, Manipulation of the angiopoietic/hemangiopoietic commitment in the avian embryo, Development 126 (1999) 617-627.

[36] S.A. Vokes, T.A. Yatskievych, R.L. Heimark, J. McMahon, A.P. McMahon, P.B. Antin, P.A. Krieg, Hedgehog signaling is essential for endothelial tube formation during vasculogenesis, Development 131 (2004) 4371-4380.

[37] M. Peeters, K. Ottersbach, K. Bollerot, C. Orelio, M. de Bruijn, M. Wijgerde, E. Dzierzak, Ventral embryonic tissues and Hedgehog proteins induce early AGM hematopoietic stem cell development, Development 136 (2009) 2613-2621.

[38] G. Swiers, C. Rode, E. Azzoni, M.F. de Bruijn, A short history of hemogenic endothelium, Blood Cells Mol Dis (2013).

[39] T. North, T.L. Gu, T. Stacy, Q. Wang, L. Howard, M. Binder, M. Marin-Padilla, N.A. Speck, Cbfa2 is required for the formation of intra-aortic hematopoietic clusters, Development 126 (1999) 2563-2575.

[40] T.E. North, M.F. de Bruijn, T. Stacy, L. Talebian, E. Lind, C. Robin, M. Binder, E. Dzierzak, N.A. Speck, Runx1 expression marks long-term repopulating hematopoietic stem cells in the midgestation mouse embryo, Immunity 16 (2002) 661-672.

[41] M. Gering, R. Patient, Notch signalling and haematopoietic stem cell formation during embryogenesis, J Cell Physiol 222 (2010) 11-16.

[42] A. Bigas, L. Espinosa, Hematopoietic stem cells: to be or Notch to be, Blood 119 (2012) 3226-3235.

[43] A. Robert-Moreno, L. Espinosa, J.L. de la Pompa, A. Bigas, $\operatorname{RBPj}\{$ kappa $\}$-dependent Notch function regulates Gata2 and is essential for the formation of intra-embryonic hematopoietic cells, Development 132 (2005) 1117-1126.

[44] A. Bigas, J. Guiu, L. Gama-Norton, Notch and Wnt signaling in the emergence of hematopoietic stem cells, Blood Cells Mol Dis 51 (2013) 264-270.

[45] A. Robert-Moreno, L. Espinosa, M.J. Sanchez, J.L. de la Pompa, A. Bigas, The notch pathway positively regulates programmed cell death during erythroid differentiation, Leukemia 21 (2007) 14961503.

[46] R.B. Lorsbach, J. Moore, S.O. Ang, W. Sun, N. Lenny, J.R. Downing, Role of RUNX1 in adult hematopoiesis: analysis of RUNX1-IRES-GFP knock-in mice reveals differential lineage expression, Blood 103 (2004) 2522-2529.

[47] R.N. Wilkinson, C. Pouget, M. Gering, A.J. Russell, S.G. Davies, D. Kimelman, R. Patient, Hedgehog and Bmp polarize hematopoietic stem cell emergence in the zebrafish dorsal aorta, Dev Cell 16 (2009) 909-916.

[48] C. Durand, C. Robin, K. Bollerot, M.H. Baron, K. Ottersbach, E. Dzierzak, Embryonic stromal clones reveal developmental regulators of definitive hematopoietic stem cells, Proc Natl Acad Sci U S A 104 (2007) 20838-20843.

[49] C.J. Marshall, C. Kinnon, A.J. Thrasher, Polarized expression of bone morphogenetic protein-4 in the human aorta-gonad-mesonephros region, Blood 96 (2000) 1591-1593.

[50] X. Guo, X.F. Wang, Signaling cross-talk between TGF-beta/BMP and other pathways, Cell research 19 (2009) 71-88.

[51] J.E. Pimanda, I.J. Donaldson, M.F. de Bruijn, S. Kinston, K. Knezevic, L. Huckle, S. Piltz, J.R. Landry, A.R. Green, D. Tannahill, B. Gottgens, The SCL transcriptional network and BMP signaling pathway interact to regulate RUNX1 activity, Proc Natl Acad Sci U S A 104 (2007) 840-845. 
[52] M.J. Goumans, G. Valdimarsdottir, S. Itoh, A. Rosendahl, P. Sideras, P. ten Dijke, Balancing the activation state of the endothelium via two distinct TGF-beta type I receptors, EMBO J 21 (2002) 17431753. 
A

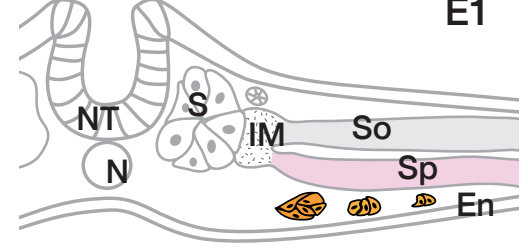

B

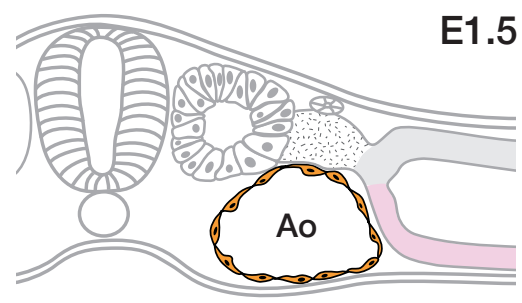

C

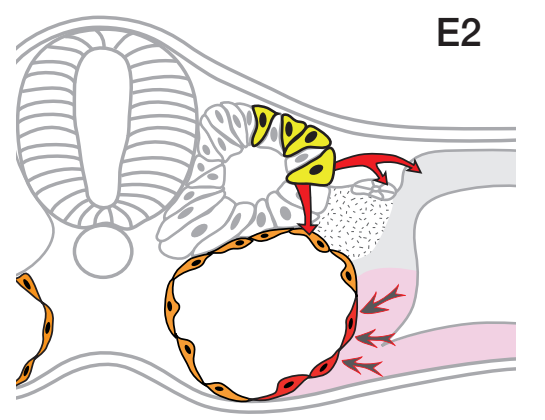

D

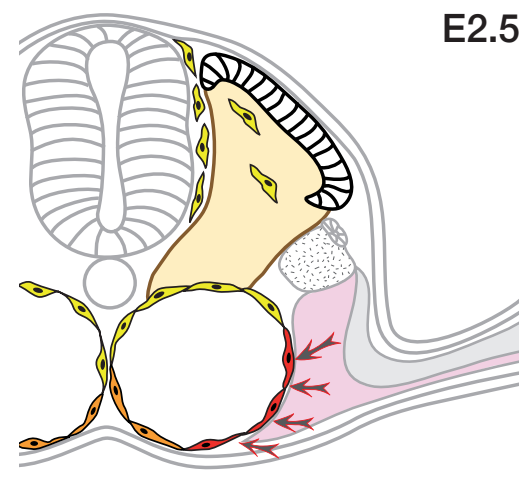

E

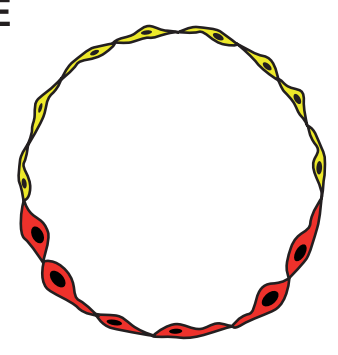

$\mathrm{F}$

notch1/jag1,2/dll4

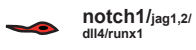

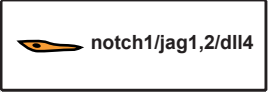

\section{E3 early}

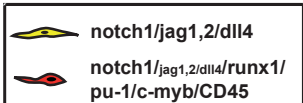

E3 late

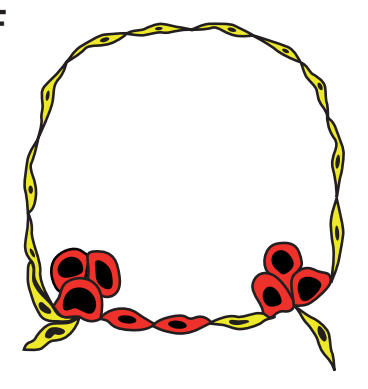

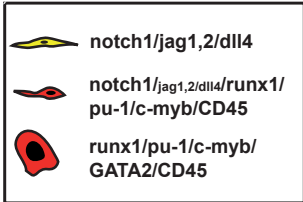

notch1/jag1,2/dII4 